\title{
A Case of Tuberous Sclerosis Complex with Lymphangioleiomyomatosis and Renal Cell Carcinoma
}

This article was published in the following Dove Press journal: OncoTargets and Therapy

\author{
Yun $\mathrm{Wu}^{*}$ \\ Hongru Li* \\ Xiaoli Yu \\ Ming Lin \\ Yusheng Chen \\ Department of Respiratory Medicine, \\ Fujian Provincial Hospital, Fujian Medical \\ University, Fuzhou, Fujian 35000I, \\ People's Republic of China \\ *These authors contributed equally to \\ this work
}

\begin{abstract}
Lymphangioleiomyomatosis (LAM) is a rare disease affecting young women, which occurs sporadically or in patients with tuberous sclerosis complex (TSC). The main manifestations of TSC in the kidney include cysts and angiomyolipoma (AML). Although renal cell carcinoma (RCC) is not a manifestation of TSC, it has a $2-4 \%$ incidence in TSC patients. Furthermore, LAM is rare in patients with RCC. Herein, we present a case of a 40-year-old woman with LAM and RCC in the right kidney. We checked for mutations in the TSC1 and TSC2 genes from both blood and kidney lesions and found a heterozygous mutation of c.1717-30G $>$ A in intron 16 of TSC2 gene. In TSC patients, the diagnosis of RCC is challenging because the cancer is rare, and it is often difficult to distinguish it from AML with conventional imaging techniques. Therefore, it is recommended that patients with TSC undergo renal imaging follow-ups annually for kidney masses.
\end{abstract}

Keywords: tuberous sclerosis complex, TSC, lymphangioleiomyomatosis, LAM, renal cell carcinoma, RCC, TSC1, TSC2

\section{Introduction}

Lymphangioleiomyomatosis (LAM) is a rare neoplastic disease affecting women and is associated with progressive cystic lung destruction, chylous pleural effusions and/or ascites, renal angiomyolipomas, and lymphangioleiomyomas. ${ }^{1}$ LAM occurs sporadically or in patients with tuberous sclerosis complex (TSC). ${ }^{2}$ The main manifestations of TSC in the kidney include cysts and angiomyolipoma (AML). Although renal cell carcinoma (RCC) is not a manifestation of TSC, its incidence in TSC patients is reported to be about 2-4\%; furthermore, its appearance with LAM is rare. ${ }^{3}$ Therefore, herein we present a rare case of a TSC patient with LAM and $\mathrm{RCC}$ diagnosed by radio imaging, histology, and gene assay.

\section{Case Presentation}

A 40-year-old woman presented with a 1-year history of dry cough. She had a positive history of shortness of breath, particularly when climbing stairs. She did not have a history of seizures. She was diagnosed with angiomyolipoma (about $4.9 \mathrm{~cm} \times 2.3 \mathrm{~cm}$ ) in the left kidney 11 years ago, which was excised to prevent spontaneous rupture. She denied any history of smoking cigarettes. Her father died of lung cancer at age 65 years. Her mother was 64 years old and had no clinical features of TSC. She has had 2 parous experiences with no complications. Her son is 15 years old and her daughter is 11 years old; both have no TSC manifestations.
Correspondence: Yusheng Chen Department of Respiratory Medicine, Fujian Provincial Hospital, Fujian Medical University, Fuzhou, Fujian 35000I, People's Republic of China Email cysktz@I63.com
OncoTargets and Therapy 2020:13 |242I-12426

$|242|$ 
She was clinically oriented with normal intelligence. No skin lesion was noted, and there was no pneumothorax. Her vital signs were stable on admission and her oxygen saturation was $96 \%\left(\mathrm{FiO}_{2}, 21 \%\right)$. We performed laboratory tests, including routine blood examination, liver and kidney functions, coagulation function, and routine urine tests; the results of all these tests were normal. Other findings included vascular endothelial growth factor-D (VEGF-D), $425 \mathrm{pg} / \mathrm{mL}$; antinuclear antibodies, $11000(+)$; and negative anti-neutrophil cytoplasmic antibodies. Arterial blood gas analysis showed a partial pressure of oxygen of $86.5 \mathrm{mmHg}$ and a $\mathrm{pCO}_{2}$ of $34.6 \mathrm{mmHg}$. Her pulmonary function tests showed a forced vital capacity (FVC) of $2.61 \mathrm{~L}(88.2 \%$ of the predicted value), forced expiratory volume in 1 second $\left(\mathrm{FEV}_{1}\right)$ of $1.55 \mathrm{~L}(61.1 \%$ of the predicted value), and $\mathrm{FEV}_{1} / \mathrm{FVC}$ ratio of $59.42 \%$.

As shown in Figure 1A, chest high-resolution computed tomography (HRCT) showed numerous thin-walled cysts within the lungs and diffuse ground-glass lung opacities, consistent with the diagnosis of LAM. As shown in Figure $1 \mathrm{~B}$, abdominal contrast-enhanced CT showed a lesion at the lower pole of the right kidney $(1.7 \mathrm{~cm} \times 1.8 \mathrm{~cm})$, which is of a non-fatty component. The lesion was well-circumscribed, with abundant blood supply. Echocardiography, electroencephalography, brain magnetic resonance imaging, and ophthalmologic evaluation revealed no abnormal findings. The patient's lung tissue was not biopsied.

Hematoxylin and eosin (H\&E) staining and immunohistochemical staining were conducted after the surgical resection of the lesion in the right kidney. As shown in Figure $2 \mathrm{~A}$ and $\mathrm{B}, \mathrm{H}$

\&E staining showed typical alveolar arrangement of cells. Figure C-F showed that the lesion was positive for CD10(+++), CA9(+++), Ki67(5\%), and Vimentin(+++) and negative for CD117, CK7, TFE3, RCC, and P504s. $\mathrm{H} \& \mathrm{E}$ staining and immunohistochemical staining confirmed the lesion to be RCC rather than AML. We checked for mutations of TSC1 and TSC2 genes from both blood and kidney lesions and found a heterozygous mutation of c. $1717-30 \mathrm{G}>\mathrm{A}$ in intron 16 of the TSC2 gene (Figures 3 and 4).

Although not showing any clinical manifestations from history and physical examination, the patient's mother, son, and daughter showed the same mutation (Figure 4). We found this mutation to be related to TSC in the ClinVar databases. ${ }^{4}$

The patient was started on oral rapamycin $1 \mathrm{mg} / \mathrm{d}$ after the surgery, approximately 1 year ago. Two months ago,
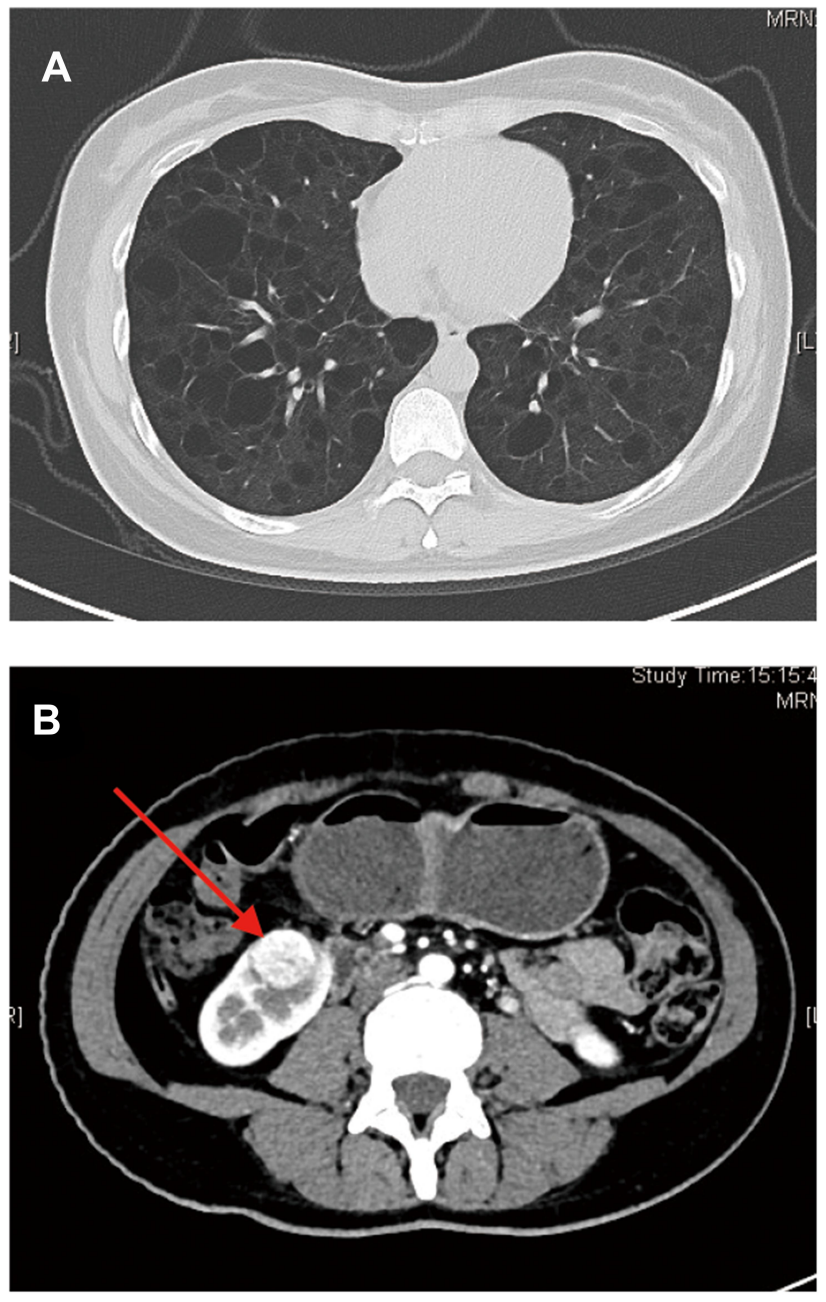

Figure I Computed tomography (CT) imaging of the chest and kidney. Notes: (A) Chest CT imaging shows numerous thin-walled cysts within the lungs and diffuse ground-glass lung opacities; (B) The abdominal enhanced CT image shows a lesion at the lower pole of the right kidney (red arrow), which is a nonfatty in nature.

she underwent pulmonary function testing and her pulmonary function had improved $\left(\mathrm{FEV}_{1}, 67.3 \%\right.$ of the predicted value; $\mathrm{FEV}_{1} / \mathrm{FVC}$ ratio, 66.31\%). Currently, the patient no longer experiences shortness of breath and there has been no tumor recurrence.

We obtained written informed consent from the patient for the publication of this case report and the accompanying images.

\section{Discussion}

This report discusses a typical LAM case that is seen in women of childbearing age, presenting with multiple thinwalled cystoid changes in the lungs on chest HRCT images. A high serum level of VEGF-D $(>800 \mathrm{pg} / \mathrm{mL})$ is diagnostic of LAM. The patient's serum level of VEGF-D 

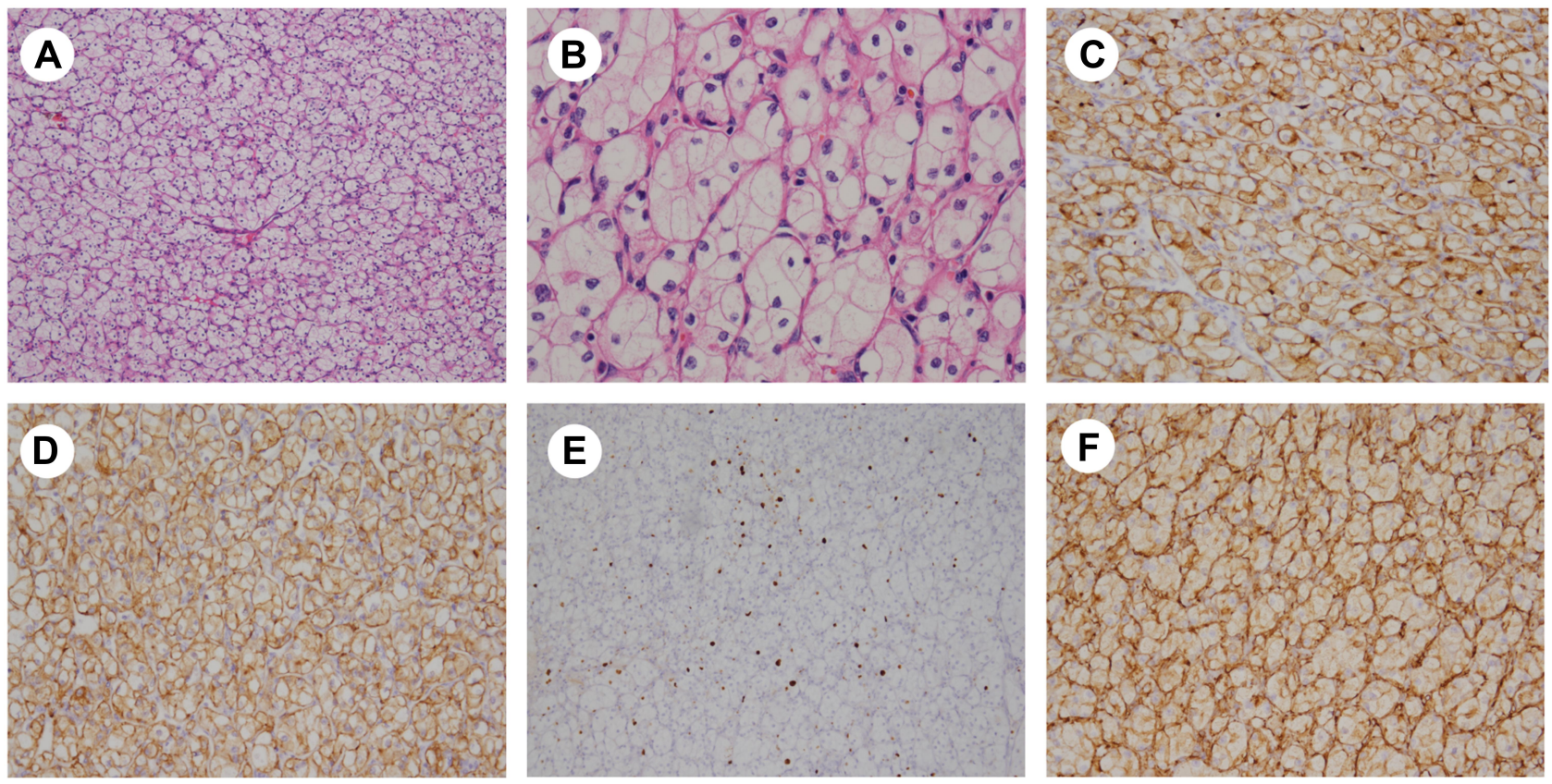

Figure 2 Histopathologic analysis.

Notes: H\&E and immunohistochemical staining of TSC-RCC (Fuhrman: CIN II). (A) H\&E I00x; (B) H\&E 400x; (C) CDI0(+++) I00x; (D) CA9(+++) I00x; (E) Ki67(5\%) 100x; (F) Vimentin(+++) 100x. H\&E staining and immunohistochemical staining confirmed the lesion to be RCC rather than an AML.

level was $435 \mathrm{pg} / \mathrm{mL}$. However, a normal VEGF-D level does not rule out the diagnosis of LAM. ${ }^{5}$ Concomitantly, a RCC was found in her right kidney, and she had a history of left renal AML surgery. According to the International Tuberous Sclerosis Complex Diagnostic Criteria, a combination of LAM and AML without other features does not meet the criteria for a definite diagnosis of TSC; however, this patient had a mutation in the TSC2 gene, which was identified as an independent diagnostic criterion. ${ }^{6}$ Furthermore, the patient's mother, son, and daughter had the same mutation in the TSC2 gene, which confirms the diagnosis of TSC regardless of the clinical findings. Although the gene mutation was located in an intron, it was likely a pathogenic mutation according to previous case reports, which may cause TSC through alternative splicing. ${ }^{7,8}$ This is further supported by the finding that the patient's RCC had the same mutation in the TSC2 gene. However, it remains unclear whether the gene mutation was related to renal cancer.

The woman had a history of AML, which is common in TSC patients. She did not have clinical symptoms of RCC, such as painless hematuria, and the CT scan, which showed a well-circumscribed lesion, was suggestive of AML. Considering the patient's history, her clinical features, and the CT scan findings, the lesion in the right kidney was considered more likely to be non-fatty AML than RCC.
The patient consented to having the lesion removed after counselling, and it was proven to be RCC based on HE and immunohistochemical findings. TSC with renal cancer is commoner than simple renal cancer in women with an earlier onset. ${ }^{9}$ In this patient, there were no significant abnormal clinical characteristics, except the isolated lesion found in the right kidney, which could easily be overlooked.

In TSC patients, the diagnosis of RCCs remains challenging because they are uncommon and often difficult to distinguish them from non-fatty AML using conventional imaging techniques alone. ${ }^{10}$ Avila et al conducted multiple CT follow-ups of 33 patients with nonfatty renal masses in LAM patients and found that 4 patients showed masses that continued to grow for four years $(>0.5 \mathrm{~cm} / \mathrm{y})$ and were later confirmed to be RCC. ${ }^{11}$ In the remaining 29 renal tumors, the diameter did not increase significantly $(<0.5 \mathrm{~cm} / \mathrm{y})$. On biopsy, nine of them were found to be nonfatty AML after the biopsy. Therefore, patients with TSC are recommended to undergo renal imaging follow-ups annually for nonfatty kidney masses. If there is a trend of continuous growth or a significant increase in the size of the mass during the follow-up period, it is recommended that a biopsy should be performed to distinguish between RCC and AML.

Rapamycin has been proven to be an effective therapy for TSC patients. Our patient presented to the hospital with dry cough; after one year of rapamycin therapy, she no 


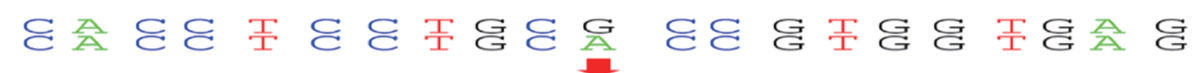

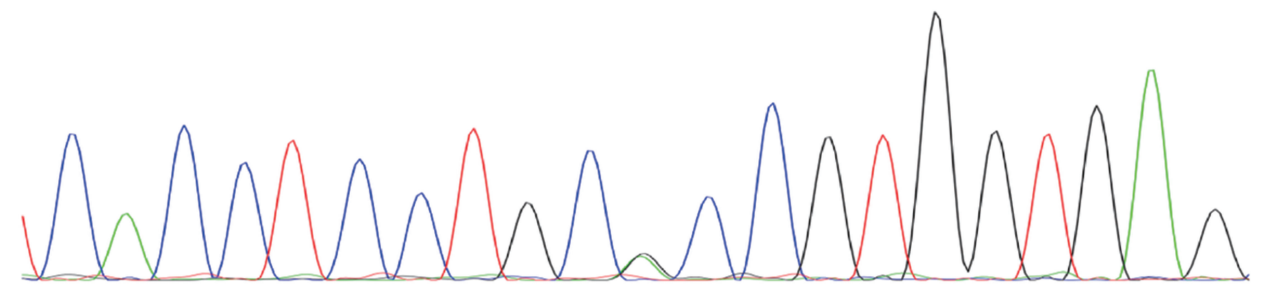

The patient

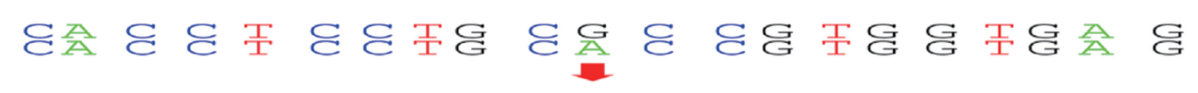

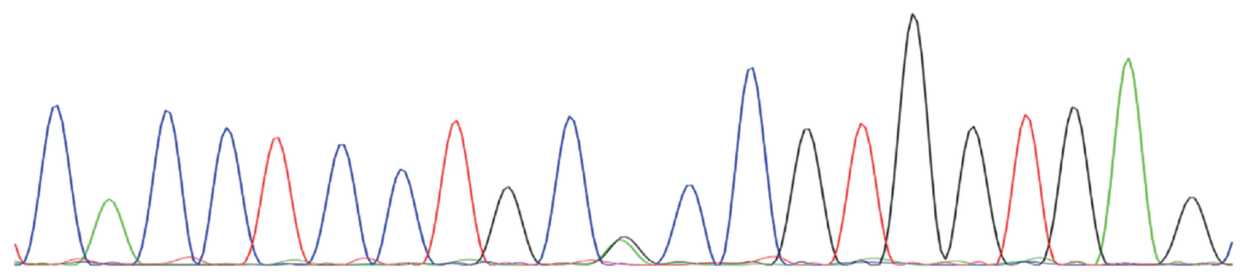

Mother of the patient

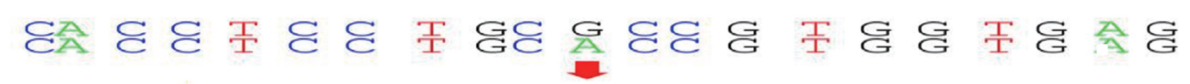

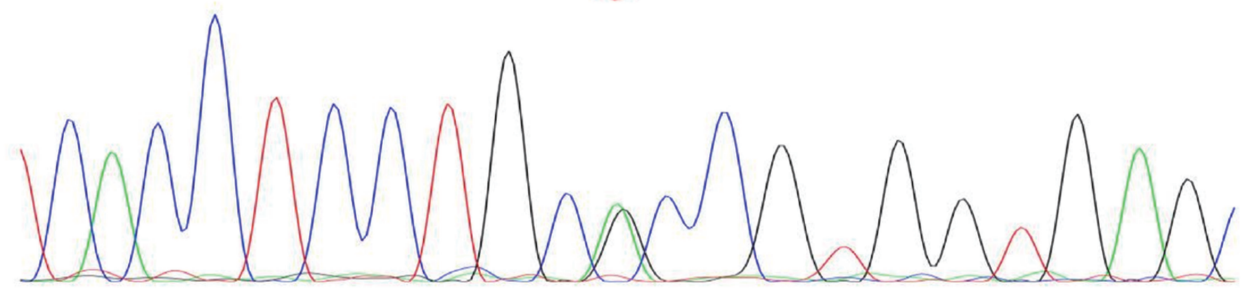

Son of the patient

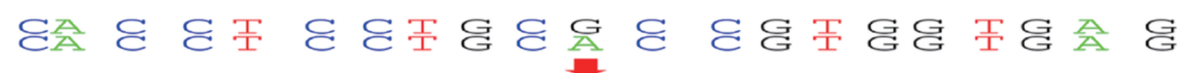

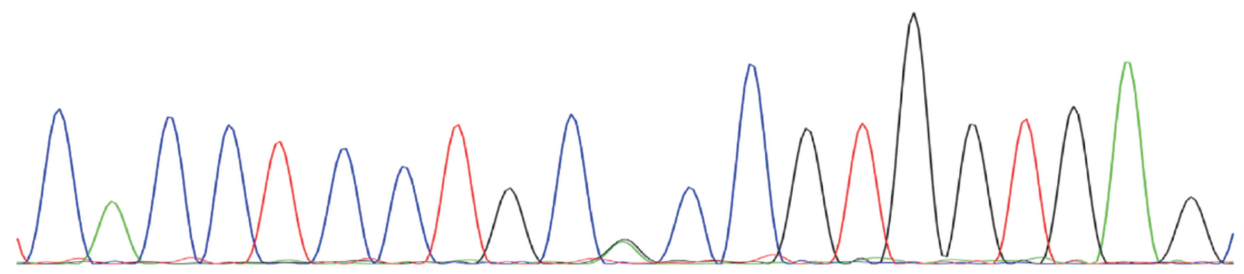

Daughter of the patient

Figure 3 Sequence chromatograms of the TSC2 gene.

Notes: A same heterozygous TSC2 mutation c. I7I7-30G>A (red arrow) is identified in the patient, her mother, son, and daughter. Although the gene mutation was located in an intron, this gene mutation is likely a pathogenic mutation according to previous case reports.

longer had shortness of breath and her pulmonary function had improved. Based on the present case, we speculate that rapamycin may be beneficial to TSC patients with RCC and LAM.
The comorbidity of RCC and LAM in patients with TSC is rare, and the diagnosis of RCC in TSC is thus challenging. We presented the case of a woman with this rare combination, which was diagnosed by relevant family 


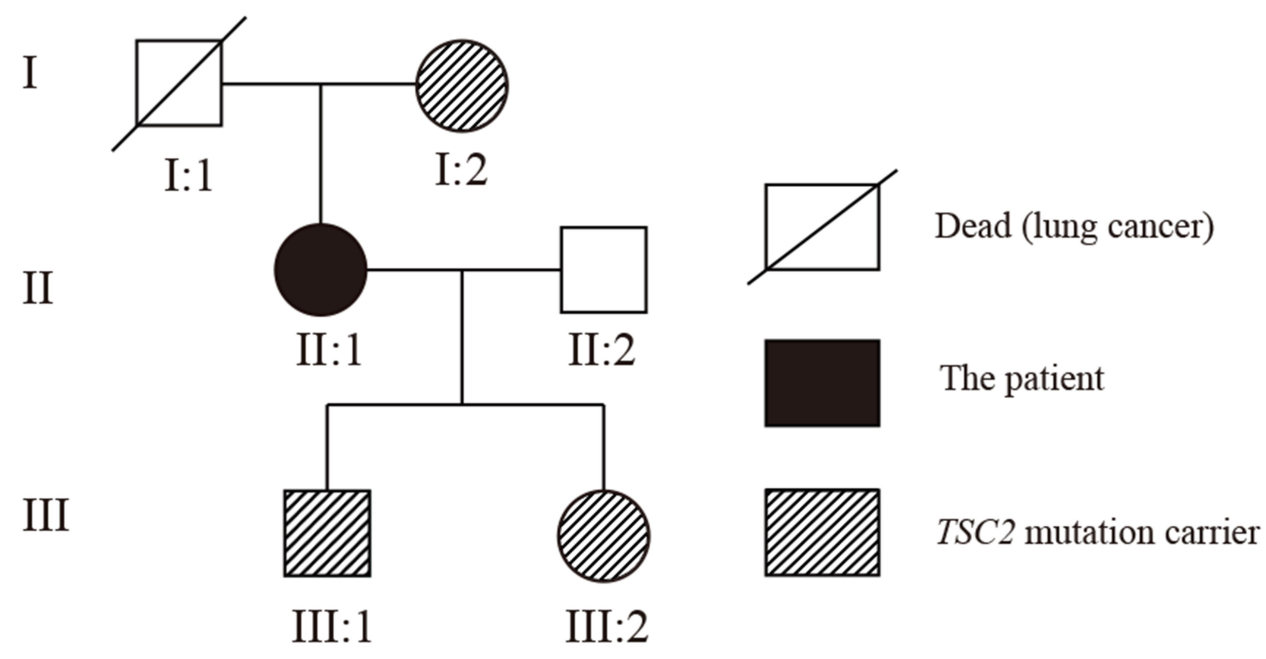

Figure 4 Pedigree for the family with a TSC2 mutation.

Notes: The patient, her mother, son, and daughter has the TSC2 gene mutation; however, only the patient is currently affected.

history, imaging, histological investigations and gene assay. She underwent surgery and did well on postoperative rapamycin therapy as evidenced by regular pulmonary function testing.

\section{Abbreviations}

LAM, lymphangioleiomyomatosis; TSC, tuberous sclerosis complex; AML, angiomyolipoma; RCC, renal cell carcinoma.

\section{Data Sharing Statement}

The data used to support the findings of this study are available from the corresponding author upon request.

\section{Ethics Approval}

The study was approved by the institutional medical ethics committee of Fujian Provincial Hospital (K2020-06-012).

\section{Consent for Publication}

Written informed consent was obtained from the patient for the publication of this case report and the accompanying images.

\section{Author Contributions}

All authors made substantial contributions to conception and design, acquisition of data, or analysis and interpretation of data; took part in drafting the article or revising it critically for important intellectual content; agreed to submit to the current journal; gave final approval of the version to be published; and agree to be accountable for all aspects of the work.

\section{Funding}

This work was financially supported by grants from The National Key Research and Development Program of China (2016YFC0901502). The funders had no role in the study design, data collection and analysis, decision to publish, or preparation of the manuscript.

\section{Disclosure}

The authors report no conflicts of interest in this work.

\section{References}

1. Ryu JH, Moss J, Beck GJ, et al. The NHLBI lymphangioleiomyomatosis registry: characteristics of 230 patients at enrollment. Am $J$ Respir Crit Care Med. 2006;173(1):105-111. doi:10.1164/rccm. 200409-12980C

2. McCormack FX. Lymphangioleiomyomatosis: a clinical update. Chest. 2008;133(2):507-516. doi:10.1378/chest.07-0898

3. Yang P, Cornejo KM, Sadow PM, et al. Renal cell carcinoma in tuberous sclerosis complex. Am J Surg Pathol. 2014;38(7):895-909. doi:10.1097/PAS.0000000000000237

4. Hung -C-C, Su Y-N, Chien S-C, et al. Molecular and clinical analyses of 84 patients with tuberous sclerosis complex. BMC Medical Genetics. 2006;7(1):72. doi:10.1186/1471-2350-7-72

5. Feemster LC, Lyons PG, Chatterjee RS, et al. Summary for Clinicians: lymphangioleiomyomatosis Diagnosis and Management Clinical Practice Guideline. Ann Am Thorac Soc. 2017;14 (7):1073-1075.

6. Northrup H, Krueger DA, Northrup H. Tuberous Sclerosis Complex Diagnostic Criteria Update: recommendations of the 2012 International Tuberous Sclerosis Complex Consensus Conference. Pediatr Neurol. 2013;49(4):243-254. doi:10.1016/j.pediatrneurol.20 13.08.001

7. Mayer K, Ballhausen W, Rott H-D. Mutation screening of the entire coding regions of theTSC1 and theTSC2 gene with the protein truncation test (PTT) identifies frequent splicing defects. Hum Mutat. 1999;14(5):401-411. doi:10.1002/(SICI)1098-1004(199911)14:5<40 1::AID-HUMU6>3.0.CO;2-R 
8. Li Y, Cao J, Chen M, et al. Abnormal Neural Progenitor Cells Differentiated from Induced Pluripotent Stem Cells Partially Mimicked Development of TSC2 Neurological Abnormalities. Stem Cell Reports. 2017;8(4):883-893. doi:10.1016/j.stemcr.2017. 02.020

9. Lam HC, Siroky BJ, Henske EP. Renal disease in tuberous sclerosis complex: pathogenesis and therapy. Nat Rev Nephrol. 2018;14 (11):704-716. doi:10.1038/s41581-018-0059-6
10. Lam HC, Nijmeh J, Henske EP. New developments in the genetics and pathogenesis of tumours in tuberous sclerosis complex. J Pathol. 2017;241(2):219-225.

11. Avila NA, Dwyer AJ, Moss J. Active Surveillance of Nonfatty Renal Masses in Patients With Lymphangioleiomyomatosis: use of CT Features and Patterns of Growth to Differentiate Angiomyolipoma From Renal Cancer. AJR Am J Roentgenol. 2017;209(3):611-619. doi:10.2214/AJR.16.17530

\section{Publish your work in this journal}

OncoTargets and Therapy is an international, peer-reviewed, open access journal focusing on the pathological basis of all cancers, potential targets for therapy and treatment protocols employed to improve the management of cancer patients. The journal also focuses on the impact of management programs and new therapeutic

Submit your manuscript here: https://www.dovepress.com/oncotargets-and-therapy-journal agents and protocols on patient perspectives such as quality of life, adherence and satisfaction. The manuscript management system is completely online and includes a very quick and fair peer-review system, which is all easy to use. Visit http://www.dovepress.com/ testimonials.php to read real quotes from published authors. 